\title{
Particle generation for pharmaceutical applications using supercritical fluid technology
}

\author{
Jacques Fages*, Hubert Lochard, Jean-Jacques Letourneau, Martial Sauceau, Elisabeth Rodier \\ École des Mines d'Albi-Carmaux, Chemical Engineering Laboratory for Particulate Solids, UMR-CNRS 2392, 81013 Albi, France
}

\begin{abstract}
In the pharmaceutical industry, an even greater number of products are in the form of particulate solids. Their formation, formulation and the control of their user properties are still not well understood and mastered. Since the mid-1980s, a new method of powder generation has appeared involving crystallisation with supercritical fluids. Carbon dioxide is the most widely used solvent and its innocuity and "green" characteristics make it the best candidate for the pharmaceutical industry. Rapid Expansion of Supercritical Solutions (RESS), Supercritical Anti Solvent (SAS) and Particles from Gas Saturated Solutions (PGSS) are three families of processes which lead to the production of fine and monodisperse powders, including the possibility of controlling crystal polymorphism. For the RESS process, the sudden decompression of the fluid in which a solute has been dissolved is the driving force of nucleation. $\mathrm{CO}_{2}$ is, however, a rather feeble solvent and this is obviously the main limitation of the development of RESS. In the SAS process, $\mathrm{CO}_{2}$ acts as a non-solvent for inducing the crystallisation of a solute from an organic solution. The versatility of SAS (there is always a proper solvent-antisolvent couple for the studied solute) ensures future developments for very different types of materials. PGSS uses the fact that it is much easier to dissolve $\mathrm{CO}_{2}$ in organic solutions (or melted compounds) than the contrary. It presents very promising perspectives of industrial development. After almost 20 years of active research, and more than 10 years of process development, this technology is reaching maturity, and very soon commercial drug produced by these techniques are likely to appear.
\end{abstract}

Keywords: Particle generation; Pharmaceutical application; Supercritical fluid technology

\section{Introduction}

About two thirds of the products used in the pharmaceutical industry are in the form of particulate solids [1]. Consequently, a lot of effort has been put into research in particle generation processes. The standard processes, crushing/milling and crystallisation/precipitation are still the most used. However, supercritical fluid (SCF) technology presents a new and interesting route for particle formation, which avoids most of the drawbacks of the traditional methods. Supercritical processes give micro- or even nanoparticles with narrow size distribution, and can also be used to achieve microencapsulation, surface coating of an active substance particle with a polymer or co-crystallisation with 25

* Corresponding author. Tel.: +33-563-49-31-41; fax: +33-563-49-30-

E-mail address: Jacques.Fages@enstimac.fr (J. Fages). excipients or host molecules like cyclodextrins. In addition, several other advantages can be noted depending on the chosen process configuration:

- high purity of products;

- control of crystal polymorphism;

- possibility of processing thermolabile molecules;

- single-step process;

- easy downstream processing;

- environmentally acceptable technology.

Among these advantages, most of them due to the use of carbon dioxide $\left(\mathrm{CO}_{2}\right)$ of which properties of nontoxicity and mild critical conditions make it an ideal substitute to organic solvents. Moreover, $\mathrm{CO}_{2}$ is gaseous at ambient conditions, which simplifies the problem of solvent residues.

The bioavailability of pharmaceutical molecules depends on their absorption by the gastrointestinal tract, which is 


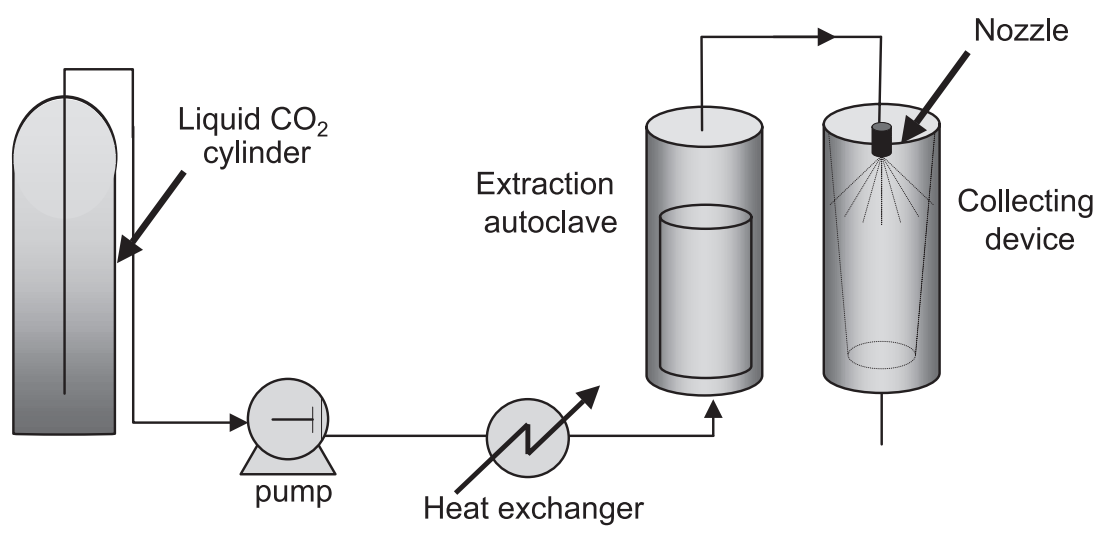

Fig. 1. Schematic flow diagram of the RESS process.

governed by their dissolution and membrane permeation rates. Micronisation techniques, and especially those using $\mathrm{SC}-\mathrm{CO}_{2}$, which lead to an increase in the specific surface area (typically several tens of $\mathrm{m}^{2} \mathrm{~g}^{-1}$ for a nonporous solid), can significantly contribute to this improvement in bioavailability. The development of dry powder inhalers (DPI) in which the drug is directly delivered to the lungs requires also fine powders with a mean particle size in the range of 2 to $5 \mu \mathrm{m}$.

Microencapsulation, coating and formation of composite particles are also extremely desirable for controlled delivery systems. Here again, supercritical technology may bring new solutions to old problems.

Rapid Expansion of a Supercritical Solutions (RESS), Supercritical Anti-Solvent (SAS) and, to a lesser extent, Particles from Gas Saturated Solutions (PGSS) are the most studied processes. It is known that RESS can be used with $\mathrm{CO}_{2}$-soluble molecules, while SAS can process nonsoluble molecules. However, the choice between these two methods is not so simple in reality. To choose among these processes and the large domain of possible operating conditions requires a good knowledge of the phase equilibria thermodynamics.

\section{The RESS process}

\subsection{A two-step process: solubilisation and particle formation}

RESS is a two-step process: after having solubilised a substance in an SCF, the mixture is suddenly depressurised in a nozzle causing fast nucleation and fine particle generation (Fig. 1). In designing a RESS process, it is necessary to have the best possible understanding of what happens upstream from the nozzle, that is to say during the extraction step. Therefore, it is important either to collect data from the literature when they exist, or to perform experiments or modelling, about the solute solubility in the SCF [2,3].

The key parameters of the extraction step are obviously the operating $T$ and $P$. The flow rate of the fluid may also play an important role since a thermodynamic equilibrium may or may not be reached in the extraction autoclave. In fact, the kinetics of the dissolution must not be neglected and diffusion limitations can occur. Additional problems may be encountered when the solid to be extracted is not a pure component. Indeed, fractionation of the load can lead to a variation in the composition of the particles upon depressurisation.

To understand what will happen during fluid expansion, phase diagrams are very useful. Fig. 2 shows the $(P, T)$ and $(P, h)$ diagrams for pure $\mathrm{CO}_{2}$. Phase diagrams and results presented in this article have been simulated using multiphase equilibrium calculations. The fluid phases are modelled using the Peng-Robinson's equation of state with various mixing rules. What can be noticed is that these phase equilibria involve pure solid constituents. The pure solid chemical potential used in the model does not require the value of the sublimation pressure. When the expressions of the required properties are unknown, which is the case for most of pharmaceutical molecules, this pure solid chemical potential expression is based on the measurements made with a differential scanning calorimeter of the melting point of the solid. The fugacity coefficient $\varphi_{i, \mathrm{~S}}^{(\text {pure })}$ in this chemical potential is the following:

$$
\begin{aligned}
& \ln \varphi_{i, \mathrm{~S}}^{\text {(pure) }}(P, T) \\
& \left.=\ln \varphi_{i, \mathrm{~L}}^{(\text {pure })}(P, T)+1-\frac{T_{i}^{(\mathrm{fus})}}{T}\right) \frac{\Delta h_{i}^{(\mathrm{fus})}\left(P^{(\mathrm{std})}\right)}{R T_{i}^{(\mathrm{fus})}} \\
& \quad-\int_{P^{(\text {std })}}^{P} \frac{\Delta-\mathrm{S} v_{i}(u, T)}{R T} \mathrm{~d} u-\frac{1}{R T} \int_{T_{i}^{(\text {fus })}}^{T}\left(1-\frac{T}{u}\right) \\
& \quad \times \underset{\mathrm{L}-\mathrm{S}}{\Delta} c_{P_{i}}\left(P^{(\mathrm{std})}, u\right) \mathrm{d} u
\end{aligned}
$$

where: $\underset{\mathrm{L}-\mathrm{S}}{\Delta} v_{i}(P, T)=v_{i, \mathrm{~L}}^{\text {(pure) }}(P, T)-v_{i, \mathrm{~S}}^{\text {(pure) }}(P, T)$ is the difference between pure liquid and pure solid molar volume and $\underset{\mathrm{L}-\mathrm{S}}{\Delta} c_{P_{i}}\left(P^{(\text {std })}, T\right)=c_{P_{i}, \mathrm{~L}}^{\text {(pure) }}(T)-c_{P_{i}, \mathrm{~S}}^{\text {(pure) }}(T)$ (with $P^{(\mathrm{std})}=1$ atm) is the difference between pure liquid and pure solid molar heat capacities. More details on the numerical calculation of these phase equilibria are given elsewhere [4]. 

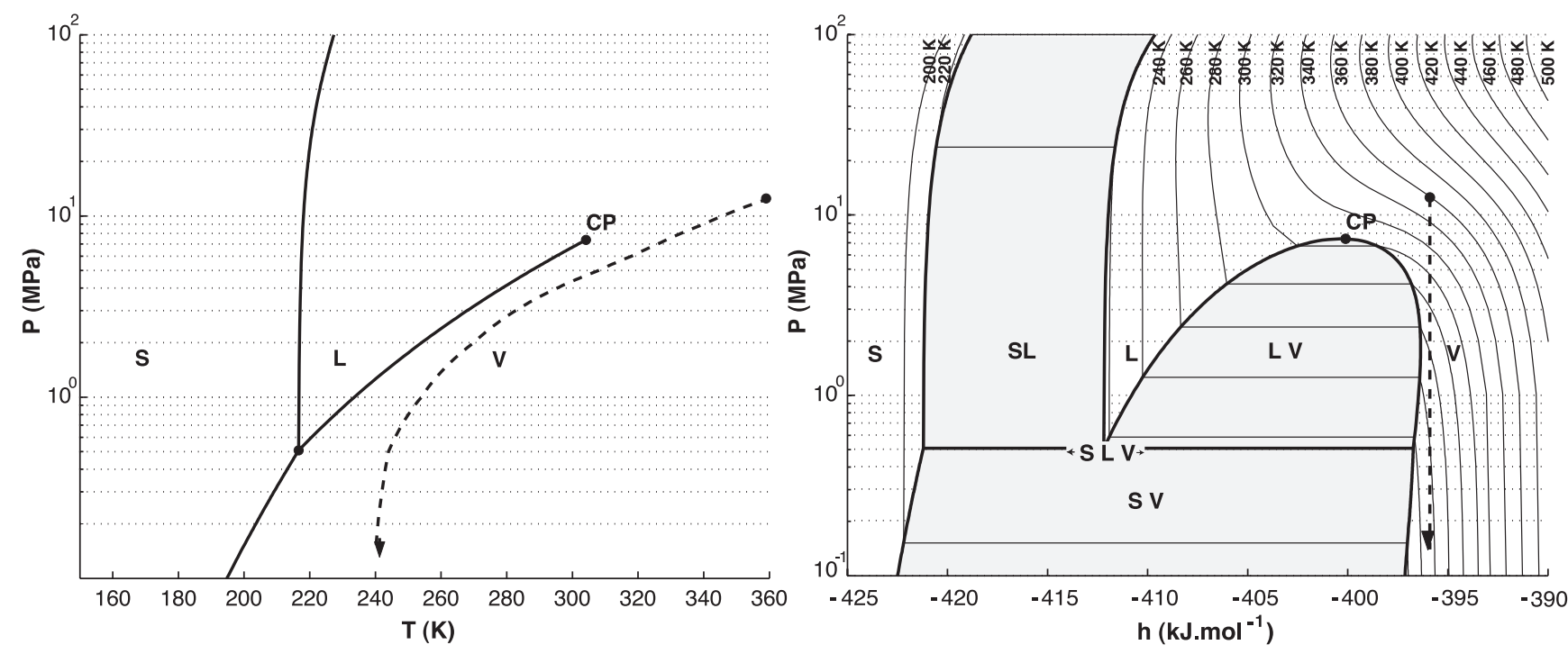

Fig. 2. Isenthalpic depressurisation of pure $\mathrm{CO}_{2}$ on $P, T$ (pressure, temperature) and $P, h$ (pressure, molar enthalpy) phase diagrams.

An acceptable assumption is to consider that the depressurisation is an isenthalpic process provided the variation of kinetic energy can be neglected. This is, however, a better assumption for capillaries than for plain orifices [5]. Therefore, the expansion step will be a vertical line (dashed arrow) on the $(P, h)$ diagram. Such a drop in pressure implies an important decrease in temperature, as shown in Fig. 2. In the example shown, the starting point, as well as the arrival point, are chosen to be in the single-phase zone of the diagram. However, to ensure such conditions, the temperature before expansion must be high $(360 \mathrm{~K}$ in the example given), which may be incompatible with the stability of the extracted solute. A lower pre-expansion temperature may lead to a condensation or freezing of the SCF (the triple point of $\mathrm{CO}_{2}$ is at $0.52 \mathrm{MPa}$ ).

To compensate this drop in temperature and to avoid potential problems due to clogging, a heated nozzle or a heating device just upstream from the nozzle can be used. The post-expansion pressure is also of the utmost importance.

\subsection{The mechanisms of particle formation: influence of supersaturation, temperature, pressure and hydrodynamics}

Nucleation of the solute occurs during the sudden expansion of the solution and is due to the mechanical perturbation, which propagates at the speed of sound leading to very uniform conditions [6] and therefore to a very narrow particle size distribution. Indeed, the expansion time depends not only on the speed of sound (which varies with the operating conditions and the nature of the fluid) but also on the length of the capillary nozzle. However, an approximation of between $10^{-4}$ to $10^{-6} \mathrm{~s}$ has been proposed. It is during this very short period of time that solubility falls by several orders of magnitude, leading to large supersaturation ratios. The driving force of the nucleation process is the difference between the chemical potential of the solute in both phases (fluid and solid), which is related to the activity of the solute in the solution at equilibrium. This supersaturation ratio, noted $S$, is defined as the concentration of the solute divided by the solubility (the concentration at saturation) provided the activity coefficients can be assumed to be close to 1 which is the case for low solubilities. $S$ comes from the drop in density, which is very large especially near the critical point. The value of the enhancement factor (ratio of the solubility of a given solid in SCF over the theoretical solubility in an ideal gas) can be as high as $10^{5}$ or $10^{6}$. These values give an idea of the large supersaturation ratios obtained, provided the fluid after expansion can be considered to be an ideal gas. This is the case when the expansion pressure is close to atmospheric pressure.

The rate of nucleation as well as the inverse of the nuclei size increase with $S$. Very high values of $S$ will therefore give a very large number of small nuclei. One possible way to control particle size is therefore to fine-tune the supersaturation by varying the drop in pressure through the nozzle and/or the solute concentration in the fluid. It must be noted, however, that several authors mention that some growth may occur downstream to the nozzle leading to larger particles than expected [7].

For a better control of the process, it may be advantageous to limit the depressurisation and to have a pressure of a few MPa in the expansion vessel. This will diminish the supersaturation ratio but the recycling of the fluid might be facilitated. In fact, relatively small changes in pressure may cause a dramatic fall in density and therefore in solubility. Again, the $(h, P)$ diagram shown in Fig. 2 is of great help to determine which conditions can be used.

\subsection{The limits of the RESS process}

The most obvious drawback of RESS is the fact that several families of molecules are not soluble in $\mathrm{CO}_{2}$. One 

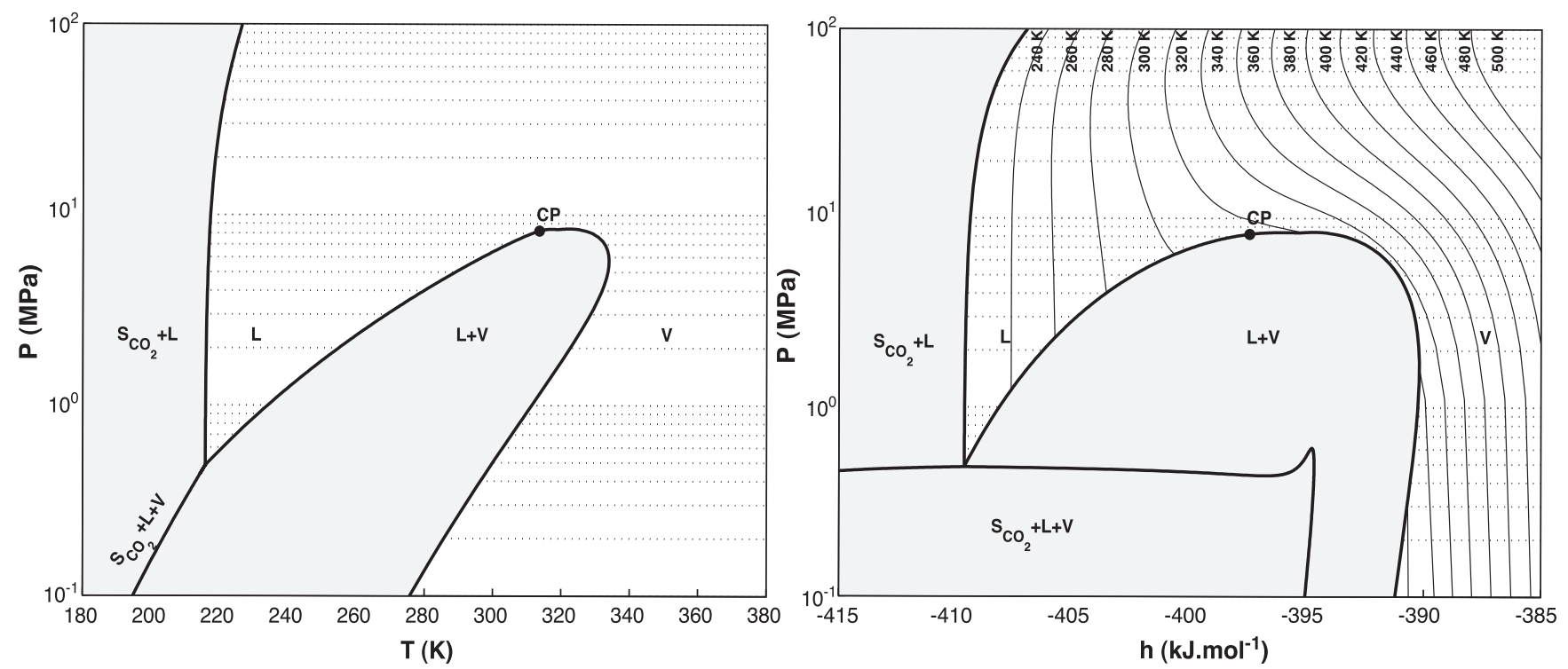

Fig. 3. $P, T$ and $P, h$ phase diagrams of a $\mathrm{CO}_{2}-$ ethanol mixture. Mole fractions: $\mathrm{CO}_{2}=98 \%$ and ethanol $=2 \%(\mathrm{calculation}$ : PR EOS + Eq. (1)).

way to overcome this problem is to change the supercritical fluid. However, this is seldom possible since the few other candidate molecules $\left(\mathrm{N}_{2} \mathrm{O}\right.$, light hydrocarbons,...) are much more hazardous and less environmental friendly than $\mathrm{CO}_{2}[8]$.

Another possibility is to use the RESS process with a cosolvent being previously dissolved in the $\mathrm{CO}_{2}$. In this case, the advantage of not using any chemical solvent is lost, but this could be attenuated by using low-toxicity solvents like acetone or ethanol.

The phase diagrams of the binary mixture are different from the pure component diagrams. As an example, the $(P$, $T)$ and $(P, h)$ diagrams for the mixture $98 \% \mathrm{CO}_{2}-2 \%$ ethanol shows the very broad two-phase envelope, which generally must be avoided (Fig. 3). This has to be taken into account when determining the operating conditions. The maximum mole fraction has to be determined to maintain a single-phase fluid after depressurisation.

As an example, the isenthalpic depressurisation of a $\mathrm{CO}_{2}$-ethanol mixture with 5\% ethanol mole fraction from $20 \mathrm{MPa}$ and $373 \mathrm{~K}$ to a pressure of $0.1 \mathrm{MPa}$ leads to the following thermodynamical equilibrium: $T=237.2 \mathrm{~K}, 5 \%$ molar of the mixture is liquid with a $97.8 \%$ mole fraction in ethanol. The particles generated under such operating conditions might be dissolved by the liquid phase that appears.

The choice of the proper co-solvent is not trivial and the two-by-two affinity of supercritical fluid, co-solvent and solute are to be considered. A mixture of co-solvents may also be used. Moreover, it is not possible to predict the cosolvent effect from the solubility of the solute in the liquid co-solvent. Therefore, solubility measurements of the solid in the mixture $\mathrm{SCF}$-co-solvent are highly recommended in order to understand the co-solvent effect in terms of molecular interactions [9].

\subsection{Applications and perspectives}

A lot of pharmaceutical products have been processed using the RESS or a RESS-derived process. Most of the published works deal with the production of single-compound particles including antibiotics, statins (anti-cholesterol drugs), sterols,.... For instance, Charoenchaitrakool et al. [10] enhanced the dissolution rate of ibuprofen with RESS. Several reviews have been devoted to these applications $[11,12]$. In addition, RESS is also usable for encapsulation processes in order to form composite particles. $\mathrm{CO}_{2}$-soluble polymers, like poly-lactic acid (PLA), are good candidates for such particle generation, as in the work of Kim et al. [13] with naproxen. With less $\mathrm{CO}_{2}$-soluble polymers like polyethylene glycol (PEG), RESS may still be usable provided a proper co-solvent can be added in the SCF. Mishima et al. [14] used this property to encapsulate several proteins in PEG. Depending on the respective solubilities of the substance to be encapsulated and of the polymer to be used, the choice of a particular process can be made. In most of the cases, a co-precipitation of two (if not more) compounds must be achieved. Microspheres, microcapsules or liposomes can thus be obtained.

Several processes have been proposed for coating preexisting microparticles. For instance, Richard et al. [15] described a process in which a $\mathrm{CO}_{2}$-soluble polymer is deposited onto particles of paracetamol by a gentle depressurisation of the autoclave. Tsutsumi et al. [16] combined the fluidized-bed technology with RESS to coat catalyst particles with paraffin.

In conclusion, RESS is a simple process usable with molecules having good or fair $\mathrm{CO}_{2}$-solubility. However, a low solubility can in some instances be accepted or overcome. Appropriate tuning of the fluid density and the use of an adequate co-solvent are two powerful tools which use 


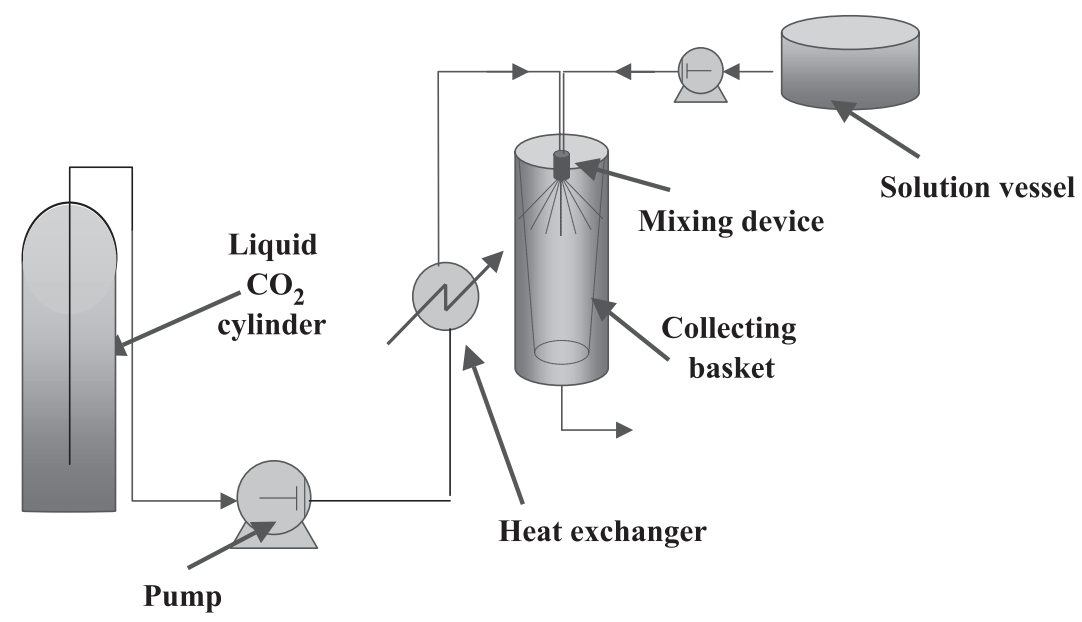

Fig. 4. Schematic flow diagram of the SAS process.

may completely change the fate of the industrial use of RESS for a specific molecule.

\section{The SAS process}

\subsection{Description of the process}

In the SAS process, the substance to be micronised is dissolved in a solvent. The SCF acts as an anti-solvent that causes the crystallisation of the solute. The discussion will be limited to semicontinuous processes in which solvent and anti-solvent are co-injected at the nozzle level. This type of process is sometimes referred as Aerosol Solvent Extraction System (ASES) or Solution Enhancement Dispersion by Supercritical fluids (SEDS). A schematic diagram of the process is shown in Fig. 4.

The particles are formed from the drops of the solution, which collide into the fluid phase. As the anti-solvent flow rate is generally much higher than that of the solution, one can use the $(P, h)$ diagram or alternatively the $(P, T)$ diagram to see how many phases are present at the equilibrium.

Applying this process to pharmaceutical compounds implies specific constraints. Special attention must be paid to the choice of the solvent. The choice of the solvent is based on three requirements. The first is its good miscibility with the anti-solvent $\left(\mathrm{CO}_{2}\right)$. Usual solvents like ethanol, toluene and acetone show complete mutual miscibility with $\mathrm{SC}-\mathrm{CO}_{2}$. The second is the solubility of the solid to be crystallised and the third is its human innocuity. Indeed, solvent must usually belong to class 3 (nontoxic) of the pharmaceutical guidelines. The most usual solvents that follow these requirements are ethanol, DMSO, acetone and ethyl acetate. In any case, the amount of residual solvent in the crystallised powder must not exceed 5000 ppm.

It is therefore often necessary to add a step of solvent stripping with fresh anti-solvent flow. Indeed, difficulties may be encountered to decrease the residual solvent content depending on the affinity between active compound and solvent. In some cases, the active compound itself may be extracted.

The following example illustrates the importance of the stripping step and the miscibility problem that could occur when some water is involved: a wet solid is dissolved in DMSO and precipitated with $\mathrm{CO}_{2}$ as anti-solvent. After precipitation, the fluid mixture has the following overall mass composition: $96.0 \%$ in $\mathrm{CO}_{2}, 3.6 \%$ in DMSO and $0.4 \%$ in $\mathrm{H}_{2} \mathrm{O}$. The operating conditions are $T=300 \mathrm{~K}$ and $P=20$ $\mathrm{MPa}$. Under these conditions, thermodynamical equilibrium leads to an immiscibility of the fluid mixture and a new liquid phase appears $(0.11 \%$ mass of the total mass mixture $)$ with a mass composition of $99.8 \%$ in water and $0.2 \%$ in $\mathrm{CO}_{2}$. The particles could be morphologically altered depending on their hygroscopicity. In any case, a stripping step with fresh $\mathrm{CO}_{2}$ is necessary to extract water and solvent residues.

\subsection{From droplets to particles}

In Fig. 3, the diagrams of a $98 \% \mathrm{CO}_{2}-2 \%$ ethanol mixture may correspond to a practical ratio of the antisolvent over the solution (ethanol and the solute) of about 50. These diagrams show the two-phase LV envelope, which has to be avoided in order not to have a liquid phase in the expansion chamber. Such a liquid phase might resolubilise the particles formed. However, these phase diagrams may change if one considers the ternary system (solute-solventantisolvent) as indicated by Reverchon et al. [17] with an antibiotic, rifampicin, precipitated by SAS with $\mathrm{CO}_{2}$ and DMSO.

In these semicontinuous processes, which are those most studied today because of their potential industrial applications, the mass transfer and the nucleation occur at the surface of solution droplets in a continuous flow of antisolvent. It is therefore necessary to understand the fate of 
these droplets. There is a competition between two phenomena that occur simultaneously:

- the anti-solvent effect, i.e. the dissolution of the antisolvent into the solution. The consequence is the swelling of the solution droplets;

- the evaporation of the solvent into the anti-solvent. The consequence is the shrinking of the solution droplets.

A key-question must therefore be addressed: do the solvent droplets in an anti-solvent flow swell or shrink?

Shekunov et al. [18] gave results obtained with optical methods: image analysis, laser interferometry and Particle Image Velocimetry (PIV). They showed that ethanol droplets in a supercritical $\mathrm{CO}_{2}$ turbulent flow do not swell as expected but on the contrary, seem to retract as long as the ethanol dissolves ("evaporates") in the $\mathrm{CO}_{2}$. Depending on the pressure (below or above the mixture critical point), the behaviour of the droplet changes. At low pressures (below the mixture critical pressure), the droplets of ethanol decrease regularly: nucleation and growth of crystals occur at the droplet interface leading to agglomerated particles. At higher pressures, a liquid ethanol jet appears from each droplet: nucleation occurs within the shrinking ethanol-rich droplets and the mixing regime has a great importance. No swelling has been observed whatever the operating conditions.

More recently, Rantakylä et al. [19] studied the influence of several parameters on the size of poly-lactic acid (PLA)particles formed in a semicontinuous SAS process. The mean particle size increased slightly with pressure and temperature and decreased with increased $\mathrm{CO}_{2}$ density. On the contrary, the velocity at the nozzle exit and the nozzle diameter do not influence the particle size. They concluded that the initial droplet sizes formed at the nozzle do not have an effect on the final particle size which is probably more determined by the reciprocal mass transfer between the solvent and the $\mathrm{CO}_{2}$.

Lora et al. [20] have given a simulation of a semicontinuous SAS with $\mathrm{CO}_{2}$ as anti-solvent, toluene as solvent and naphthalene and phenanthrene as solutes. They showed that at least the three following issues must be addressed: phase equilibria, hydrodynamics and mass transfer. Their calculations showed that the solvent droplets undergo a rapid swelling, followed by a slower shrinking phase. $\mathrm{CO}_{2}$ dissolution in the solvent and solvent evaporation into $\mathrm{CO}_{2}$ increase with the $\mathrm{CO}_{2}$ solubility in the solvent (toluene, acetone and DMSO have been compared). Crystallisation of the two tested solutes varies: the anti-solvent effect is sufficient to provoke phenanthrene crystallisation whatever its concentration in the initial solution. In addition, the phenanthrene crystallisation is hardly sensitive to the variation of the flow rate ratio solvent/anti-solvent. On the contrary, there is a threshold concentration for naphthalene below which it does not crystallise. This behaviour could be used for separation purposes when both solutes are present in the same solution.
Another study with the $\mathrm{CO}_{2}$-toluene system (without a third component to be crystallised) showed the dependence of droplet fate on the respective densities of the solvent and the anti-solvent [21]. When the anti-solvent density is lower than that of the solvent-the most common case-the droplets can swell, while they shrink in the opposite case. The extents of swelling (or shrinking) as well as the lifetime of the droplets are very sensitive to the operating conditions.

To optimise the particle production from the droplets, and particularly when very fine particles are expected, it is advantageous to work in the single-phase zone at high pressures where higher mass transfer and higher supersaturation ratios can be reached. In this zone, the average lifetime of a droplet is short, implying rapid mass transfer, high supersaturation ratios and finally very small particles. The knowledge of the phase equilibria of the binary system solvent-anti-solvent is compulsory before designing a semicontinuous SAS process.

To sum up, it is clear that several parameters have an influence on the size and the morphology of the particles obtained. As with the RESS process, the operating $T$ and $P$ (which determine the fluid density) upstream and downstream from the nozzle are obviously key parameters. The role of the nozzle appears to be essentially mixing and droplet formation, which is rather different from the RESS in which the main role of the nozzle is to provoke a huge drop in pressure. Nozzle geometry, which conditions the hydrodynamics of the jet, could be a less important parameter in controlling the process. In addition, the supersaturation linked with the concentration of the solute in the solution and the relative flow rates of the solution and the anti-solvent must also be taken into account.

\subsection{Influence on crystal polymorphism}

Compared to RESS processes, in which powder appears in a gaseous phase, in the SAS process the solute crystallises more often in a supercritical mixture $\mathrm{CO}_{2}$ /solvent. Its residence time in this mixture may induce transformations as dissolution-recrystallisation, solid/solid transition, .... Therefore, the residence time of the powder in crystallisation vessel may be an important parameter for the morphology of the powder finally obtained (polymorphism, aggregation,....).

An important issue in crystallisation can be the crystal polymorphism. A substance may exist in different forms at solid state. Depending on how the molecules fit together, different polymorphs can be encountered. This can be important in the quality of a given product. In the pharmaceutical field, an active substance may exhibit different activities and shelf life depending on the polymorph. The classical crystallisation techniques usually lead to a mixture of different polymorphs because of the multistep process used. It is therefore interesting to check if the supercritical crystallisation (a single-step unit) may give different results. 
Kordikowski et al. [22] worked with sulfathiazole, a compound having five different polymorphs. Using a semicontinuous SAS process with methanol and $\mathrm{CO}_{2}$, they showed that a good choice of the flow rate of methanol and the operating temperature may lead to a pure polymorph. Three pure polymorphs I, III and IV could be obtained by choosing the right temperature while the flow rate ratio methanol/ $\mathrm{CO}_{2}$ had less influence.

\subsection{Applications and perspectives}

Many pharmaceuticals have been processed using SAS and derived processes (SEDS, PCA, ASES). A very broad range of molecules can be used: antibiotics (chloramphenicol, tetracyclin, amoxycillin, ...), proteins (insulin, trypsin, lysozyme, catalase, peroxidase, ...), biopolymers (dextrane, PLA, PGA, HYAFF, inulin, ...), paracetamol, salbutamol, naproxen, ascorbic acid, ... [11].

In addition, it is noticeable than carriers, which are the main components of a pharmaceutical tablet, have also undergone such process. Disaccharides like maltose, lactose or sucrose are almost insoluble in most organic solvents but exhibit a great solubility in water. Unfortunately, water is very sparingly soluble in supercritical carbon dioxide. This problem has been overcome by Palakodaty et al. [23] who designed a triaxial nozzle. This device allows a co-injection of three different fluids: the supercritical anti-solvent, the aqueous solution of a sugar and an organic solution of an active substance. Water can be extracted with the organic solvent provided there exists a good mutual solubility of these two solvents.

SAS is also a technique that allows the co-precipitation of two different compounds. Several advantages can be obtained in terms of formulation, dissolution rate or drug release systems. Hereafter are a few published examples of co-precipitation.

Active substances are often quite large and polar, their solubility in supercritical $\mathrm{CO}_{2}$ is very low, prohibiting the use of RESS. The first major interest of SAS is that it is useable with all kinds of molecules. A very broad range of materials can be processed since there is almost always a useful "solvent-anti-solvent" couple. This explains why SAS and related processes have been the subject of an increasing interest during the last 5 years.

\section{The PGSS process}

A dense gas can be solubilised in large quantities in a liquid. This property is used in the PGSS process.

A dense gas, most often carbon dioxide, is dissolved in a first autoclave into a liquid, which can be either a solution of the crystallised compound (sometimes a suspension) or a melted solid. A gas-saturated solution is obtained, which can be further expanded through a nozzle in an expansion chamber. Generation of solid particles (or liquid droplets) is induced in this second vessel, then particles can be collected after completion of the process.

This process has been mainly used for polymers, in which high amounts of carbon dioxide can be dissolved. In addition, properties of the polymer, such as glass transition and melting temperatures or density, can be modified. If a third component is previously dissolved or suspended in the polymer, the final depressurisation may lead to polymer microspheres with an embedded substance. A difference from RESS and SAS processes lies in the fact that another property of the $\mathrm{CO}_{2}$ is used in the PGSS: by dissolving in a liquid at high pressures, $\mathrm{CO}_{2}$ reduces viscosity which may facilitate the handling of the solution.

For particle production, Knez [24] gave an overview of the main published applications: among them polymers [25], different kinds of lipids and two pharmaceuticals (nifedipine and felodipine) which have been successfully micronised with the PGSS process. Another particle generation process close to PGSS has been described by Sievers et al. [26]. It consists of the production of a dense finedroplet aerosol plume followed by a drying step. The aim of this process is to obtain fine particles usable in a dry powder inhaler form. By contacting supercritical carbon dioxide with an aqueous solution of an active substance in a "low-dead volume tee", followed by a depressurisation through a restrictor, they produced microdroplets which could be further dried in a stream of warm nitrogen. This patented process has been used with $\alpha$-lactose, the classic diluent for dry powder inhaler, albuterol sulfate and cromolyn sodium, two drugs active against asthma. Finally, fine spherical particles in the range of $0.1-3 \mu \mathrm{m}$ were obtained, making them suitable for inhalation.

\section{Conclusion}

Supercritical-assisted particle formation has made a lot of progress in the recent years. As noted by Elvassore and Kikic [27], the concepts of "clean or green chemistry" and "sustainable technology" are of great help to make pharmaceutical industrial applications of this technology closer than ever. Both RESS and SAS processes continue to undergo fundamental and applied research and have benefited from the recent years' advances. Several controversial issues still remain: the influence of operating parameters on the characteristics of the particle produced (size, morphology, polymorphism), the comprehension of the fluid dynamics, the nucleation phenomenon, the crystal growth under these specific conditions, the particle agglomeration in the jet, the method of scaling up, etc.

Because of its simplicity, RESS will remain the first process to be tested, while the universality of SAS will ensure future developments for very different types of materials. Very often, semicontinuous SAS process and RESS process can compete for pre-industrial particle generation. A thorough study, based on the phase equilibria and 
the solubility in pure $\mathrm{CO}_{2}$ and in $\mathrm{CO}_{2}+$ co-solvent within a broad range of $T$ and $P$, gives most of the required data for choosing the "right" process.

The industrial development of such processes is still hindered by the questions pending above mentioned but more likely by the lack of reference in the pharmaceutical industry. The capital cost, and the compliance with GMP guidelines are not real obstacles for this industry used to deal with these two parameters. Large-scale supercritical fluids facilities have been working for decades in the food industry and it is very probable that the pharmaceutical industry will soon use the new capabilities offered by this technology.

\section{Acknowledgements}

The first author would like to thank the International Fine Particle Research Institute (IFPRI) for authorising this publication which was in part presented at the 2001 annual meeting of this institute.

\section{References}

[1] C.J. Roberts, P.G. Debenedetti, Engineering pharmaceutical stability with amorphous solids, AIChE J. 48 (2002) 1140-1144.

[2] M. Sauceau, J. Fages, J.-J. Letourneau, D. Richon, A novel apparatus for accurate measurements of solid solubilities in supercritical phases, Ind. Eng. Chem. Res. 39 (2000) 4609-4614.

[3] M. Sauceau, J.-J. Letourneau, D. Richon, J. Fages, Solid compound solubilities in $\mathrm{SC}-\mathrm{CO}_{2}$ and cosolvent: development of enhanced density-based models, Fluid Phase Equilib. 208 (2003) 99-113.

[4] J.-J. Letourneau, E. Rodier, H. Lochard, M. Sauceau, J. Fages, High pressure phase equilibrium behaviour of naphthalene in supercritical $\mathrm{CO}_{2}$ : experimental results and calculation with Peng-Robinson equation of state, Proc. 8th Meeting on Supercritical Fluids, Bordeaux (France), vol. 2, ISASF International Society for the Advancement of Supercritical Fluids, Nancy, France, 2002, pp. 529-534.

[5] M. Weber, L.M. Russell, P.G. Debenedetti, Mathematical modeling of nucleation and growth of particles formed by the rapid expansion of a supercritical solution under subsonic conditions, J. Supercrit. Fluids 23 (2002) 65-80

[6] J. Tom, P.G. Debenedetti, Particle formation with supercritical fluids - a review, J. Aerosol Sci. 22 (1991) 555-584.

[7] X. Kwauk, P.G. Debenedetti, Mathematical modeling of aerosol formation by rapid expansion of supercritical solutions in a converging nozzle, J. Aerosol. Sci. 24 (1993) 445-469.

[8] D. Raynie, Warning concerning the use of nitrous oxide in supercritical fluid extractions, Anal. Chem. 65 (1993) 3127-3128.

[9] M. Sauceau, J.-J. Letourneau, B. Freiss, D. Richon, J. Fages, Solubility of Eflucimibe in supercritical carbon dioxide with or without a co-solvent, J. Supercrit. Fluids, (2004) in press.

[10] M. Charoenchaitrakool, F. Dehghani, N.R. Foster, H.K. Chan, Micronization by rapid expansion of supercritical solutions to enhance the dissolution rates of poorly water soluble pharmaceuticals, Ind. Eng. Chem. Res. 39 (2000) 4794-4802.

[11] J. Jung, M. Perrut, Particle design using supercritical fluids: literature and patent survey, J. Supercrit. Fluids 20 (2001) 179-219.
[12] B. Knutson, P.G. Debenedetti, J. Tom, Preparation of microparticulates using supercritical fluids, in: S. Cohen, H. Bernstein (Eds.), Microparticulate Systems for the Delivery of Proteins and Vaccines, Drugs and the Pharmaceutical Sciences Series, vol. 77, Marcel Dekker, New York, 1996, pp. 89-125.

[13] J.H. Kim, T.E. Paxton, D.L. Tomasko, Microencapsulation of naproxen using rapid expansion of supercritical solutions, Biotechnol. Prog. 12 (1996) 650-661.

[14] K. Mishima, K. Matsuyama, D. Tanabe, S. Yamauchi, T.J. Young, K.P. Johnston, Microencapsulation of proteins by rapid expansion of supercritical solution with a non-solvent, AIChE J. 46 (4) (2000) $857-865$.

[15] J. Richard, B. Pech, C. Thies, M.I. Ribeiro dos Santos, J.P. Benoit, Preparation and characterisation of sustained-release microcapsules obtained using supercritical fluid technology, Proc. 7th Meeting on Supercritical Fluids, Antibes (France), vol. 1, ISASF International Society for the Advancement of Supercritical Fluids, Nancy, France, 2000, pp. $143-146$.

[16] A. Tsutsumi, S. Nakamoto, T. Mineo, K. Yoshida, A novel fluidizedbed coating of fine particles by rapid expansion of supercritical fluid solutions, Powder Technol. 85 (1995) 275-278.

[17] E. Reverchon, I. de Marco, G. della Porta, Rifampicin microparticles production by supercritical antisolvent precipitation, Int. J. Pharmacogn. 243 (2002) 83-91.

[18] B. Shekunov, Y. Sun, E. Astracharchik, P. York, J. Baldyga, Optical characterisation and mechanism of antisolvent precipitation in turbulent flow, Proc. 7th Meeting on Supercritical Fluids, Antibes (France), vol. 1, ISASF International Society for the Advancement of Supercritical Fluids, Nancy, France, 2000, pp. 65-70.

[19] M. Rantakylä, M. Jäntti, O. Aaltonen, M. Hurme, The effect of initial drop size in the supercritical antisolvent precipitation (SAS) technique, J. Supercrit. Fluids 24 (2002) 251-263.

[20] M. Lora, A. Bertucco, I. Kikic, Simulation of the semi-continuous supercritical antisolvent recrystallization process, Ind. Eng. Chem. Res. 39 (2000) 1487-1496.

[21] J.O. Werling, P.G. Debenedetti, Numerical modeling of mass transfer in the supercritical antisolvent process: miscible conditions, J. Supercrit. Fluids 18 (2000) 11-24.

[22] A. Kordikowski, B. Shekunov, P. York, Crystallisation of sulfathiazole polymorphs using $\mathrm{CO}_{2}$, Proc. 7th Meeting on Supercritical Fluids, Antibes (France), vol. 1, ISASF International Society for the Advancement of Supercritical Fluids, Nancy, France, 2000, pp. 117-122.

[23] S. Palakodaty, P. York, J. Pritchard, Supercritical fluid processing of materials from aqueous solutions: the application of SEDS to lactose as a model substance, Pharm. Res. 15 (1998) 1835-1843.

[24] Z. Knez, Micronisation of pharmaceuticals using supercritical fluids, Proc. 7th Meeting on Supercritical Fluids, Antibes (France), vol. 1, ISASF International Society for the Advancement of Supercritical Fluids, Nancy, France, 2000, pp. 21-26.

[25] E. Weidner, R. Steiner, Z. Knez, Powder generation from polyethylene glycols with compressible fluids, in: Rudolph von Rohr P., Trepp C. (Eds.), High Pressure Chemical Engineering, Elsevier, Netherlands, 1996, pp. 223-228.

[26] R.E. Sievers, P.D. Milewski, S.P. Sellers, B.A. Miules, B.J. Korte, K.D. Kusek, G.S. Clack, B. Mioskowski, J.A. Villa, Supercritical and near-critical carbon dioxide assisted low-temperature bubble drying, Ind. Eng. Chem. Res. 39 (2000) 4831-4836.

[27] N. Elvassore, I. Kikic, Pharmaceutical processing with supercritical fluids, in: A. Bertucco, G. Vetter (Eds.), High Pressure Process Technology: Fundamentals and Applications, Elsevier, Netherlands, 2001, pp. $612-625$. 